\title{
Implementation of Multisensor Data Fusion Algorithm
}

\author{
Myat Su Nwe', Hla Myo Tun ${ }^{2}$ \\ ${ }^{1}$ Department of Mechanical Engineering, Yangon Technological University, Yangon, Republic of the Union of Myanmar \\ ${ }^{2}$ Department of Electronic Engineering, Yangon Technological University, Yangon, Republic of the Union of Myanmar
}

Email address:

myatsunwe@gmail.com (M. S. Nwe), hmyotun@gmail.com (H. M. Tun)

To cite this article:

Myat Su Nwe, Hla Myo Tun. Implementation of Multisensor Data Fusion Algorithm. International Journal of Sensors and Sensor Networks. Vol. 5, No. 4, 2017, pp. 48-53. doi: 10.11648/j.ijssn.20170504.11

Received: October 20, 2017; Accepted: November 1, 2017; Published: December 15, 2017

\begin{abstract}
Multisensor data fusion is the process of combining observations from a number of different sensors to provide a robust and complete description of an environment or process of interest. Data fusion finds wide application in many areas of robotics such as object recognition, environment mapping, and localization. This work has three parts: methods, architectures and applications. Most current data fusion methods employ probabilistic descriptions of observations and processes and use Bayes' rule to combine this information. Data fusion systems are often complex combinations of sensor devices, processing and fusion algorithms. This work provides an overview of key principles in data fusion architectures from both a hardware and algorithmic viewpoint. The applications of data fusion are pervasive in UAV and underlay the core problem of sensing, estimation and perception. The highlighted is many applications that bring out these features. The first describes a navigation or self-tracking application for an autonomous vehicle. The second describes an application in mapping and environment modeling. The essential algorithmic tools of data fusion are reasonably well established. However, the development and use of these tools in realistic robotics applications is still developing.
\end{abstract}

Keywords: Multisensor Data Fusion, Sensor Management, MATLAB, GUI, UAV

\section{Introduction}

HUMANS accept input from five sense organs and senses: touch, smell, taste, sound, and sight in different physical formats (and even the sixth sense as mystics tell us) [1-3]. By some incredible process, not yet fully understood, humans transform input from these organs within the brain into the sensation of being in some "reality." We need to feel or be assured that we are somewhere, in some coordinates, in some place, and at some time. Thus, we obtain a more complete picture of an observed scene than would have been possible otherwise (i. e., using only one sense organ or sensor). The human activities of planning, acting, investigating, market analysis, military intelligence, complex art work, complex dance sequences, creation of music, and journalism are good examples of activities that use advanced data fusion (DF) aspects and concepts that we do not yet fully understand. Perhaps, the human brain combines such data or information without using any automatic aids, because it has a powerful associative reasoning ability, evolved over thousands of years.

This is the information technology (IT) age, and in this context multisource multi-sensor information fusion (MUSSIF) encompasses the theory, methods, and tools conceived and used for exploiting synergy in information acquired from multiple sources (sensors, databases, information gathered by human senses, etc.). The resulting final understanding of the object (or a process or scene), decision, or action is in some sense better, qualitatively or quantitatively, in terms of accuracy, robustness, etc., and more intelligent than would be possible if any of these sources were used individually without such synergy exploitation. The above seems to be an accepted definition of information fusion [4-7]. In simple terms, the main objective in sensor DF is to collect measurements and sample observations from various similar or dissimilar sources and sensors, extract the required information, draw inferences, and make decisions. These derived or assessed information and deductions can be combined and fused, with intent of obtaining an enhanced status and identity of the perceived or observed object or phenomenon. This process is crucial for the survival and growth of humans and many other living creatures, and can be termed MUSSIF, which is popularly called sensor DF or even 
simply DF (of course, with derived and simpler meanings and lower level of information processing). Multi-sensor data fusion (MSDF) would primarily involve: (1) hierarchical transformations between observed parameters to generate decisions regarding the location (kinematics and even dynamics), characteristics (features and structures), and the identity of an entity; and (2) inference and interpretation (decision fusion) based on a detailed analysis of the observed scene or entity in the context of a surrounding environment and relationships to other entities.

In a target-tracking application, observations of angular direction, range, and range rate (a basic measurement level fusion of various data) are used for estimating a target's positions, velocities, and accelerations in one or more axes. This is achieved using state-estimation techniques like a Kalman filter. The observations of the target's attributes, such as radar cross section (RCS), infrared spectra, and visual image probably with fusion in mind, may be used to classify the target and assign a label for its identity. Pattern recognition techniques (for feature identification and identity determination) based on clustering algorithms and artificial neural networks (ANNs) or decision-based methods can be used for this purpose, to have more feature-based tracking. Understanding the direction and speed of the target's motion may help us to determine the intent of the target, which may also require automated reasoning or artificial intelligence using implicit and explicit information. For this purpose, knowledge-based methods leading to decision fusion can be used.

DF in a military context has the following meaning: "a multilevel, multifaceted process dealing with the detection, association, correlation, estimation, and combination of data and information from multiple sources to achieve a refined state and identity estimation, and complete and timely assessments of situation and threat." DF is very rapidly emerging as an independent discipline to be reckoned with and is finding ever-increasing applications in many biomedical, industrial automation, aerospace, and environmental engineering processes and systems, in addition to military defense applications [8-10].

The benefits of DF are more spatial coverage of the object under observation, redundancy of measurements so that they are always available for analysis, robustness of the system's performance, more accurate inferences (meaning better prediction with less uncertainty), and overall assured performance of the multisensor integrated systems. The complete process of DF involves the study of several allied disciplines: (1) signal or image processing; (2) computational and numerical techniques and algorithms; (3) information theoretical, statistical, and probabilistic methods; (4) sensor modeling, sensor management, control, and optimization; (5) neural networks, approximate reasoning, fuzzy logic systems, and genetic algorithms; (6) system identification and state-parameter estimation (least square methods including Kalman filtering); and (7) computational data base management. Several principles and techniques from these fields strengthen the analytical treatment and performance evaluation of DF fusion systems. Some of the foregoing aspects and methods are discussed in the present volume; spread over three parts [11-14].

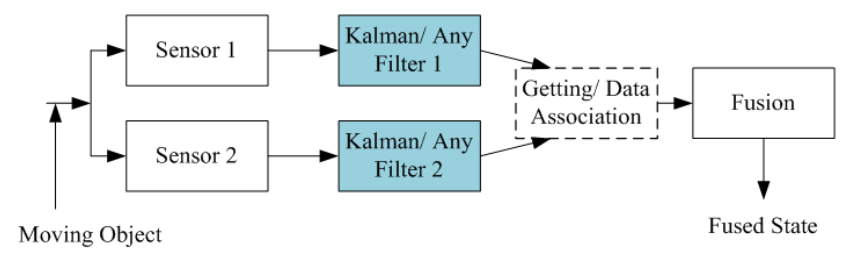

Figure 1. Sensor Data Fusion for Target Tracking.

\section{Coordinate Systems}

Coordinate systems are established to develop the navigation equations and to describe the dynamic motion of an aircraft. Aircraft navigation systems resolve the navigation equations to determine position, velocity, attitude and time (PVAT) information with respect to specific frames. Several reference coordinate systems are used in the development and design of multisensor fusion navigation systems to represent the navigation system states, aircraft kinematic parameters and navigation sensor measurements. This section reviews the definitions of some commonly used reference frames and their relationships.

A. Earth Centered Inertial (ECI) Frame $\left(x^{I}, y^{I}, z^{I}\right)$

This frame has its origin at the centre of the Earth. Its axes are non-rotating relative to the inertial space. For aircraft navigation, the ECI frame is an approximation of the Newtonian inertial frame. The $\mathrm{x}^{\mathrm{I}}$ axis is in the Earth's equatorial plane and points toward the vernal equinox. The $\mathrm{z}^{\mathrm{I}}$ axis is aligned with the Earth rotation axis. The $\mathrm{y}^{\mathrm{I}}$ axis completes the right-hand system.

B. Earth Centered Earth Fixed (ECEF) Frame $\left(x^{\mathrm{e}}, \mathrm{y}^{\mathrm{e}}, \mathrm{z}^{\mathrm{e}}\right)$

This frame is fixed to and rotating with the Earth. The ECEF frame has its origin at the Earth's centre of mass, as shown in Figure 2 (a). This is a right-hand Cartesian coordinate system. The $z^{e}$ axis is the Earth's rotation axis and points towards the direction of the Conventional Terrestrial Pole (CTP) for polar motion, as defined by BIH on the basis of the coordinates adopted for the BIH stations. The $\mathrm{x}^{\mathrm{e}}$ axis lies in the Earth's equatorial plane and points the intersection of the CTP's equator and the reference meridian being the zero meridian defined by the $\mathrm{BIH}$ on the basis of the coordinates adopted for the BIH stations. The $\mathrm{y}^{\mathrm{e}}$ axis realizes the right-hand system.

\section{Navigation Frame $\left(\mathrm{x}^{\mathrm{n}}, \mathrm{y}^{\mathrm{n}}, \mathrm{z}^{\mathrm{n}}\right)$}

This frame is attached to the aircraft and has its origin at the aircraft centre of gravity, as shown in Figure 2 (a) (b). The $z^{n}$ axis points down perpendicular to the reference ellipsoid. The $\mathrm{x}^{\mathrm{n}}$ and $\mathrm{y}^{\mathrm{n}}$ axes lie in a plane tangent to the reference ellipsoid. Therefore, the navigation frame is a local level frame and a Cartesian coordinate system. This navigation frame is a north-slaved frame if the $\mathrm{x}^{\mathrm{e}}$ axis points north and the ye axis points east, and is generally referred to as a North-East-Down (NED) frame. It is known as the wander azimuth frame $\left(\mathrm{x}^{\mathrm{w}}\right.$, $\left.\mathrm{y}^{\mathrm{w}}, \mathrm{z}^{\mathrm{w}}\right)$ if the $\mathrm{x}^{\mathrm{n}}-\mathrm{y}^{\mathrm{n}}$ plane is allowed to rotate freely about the 
$\mathrm{z}^{\mathrm{n}}$ axis. The wander azimuth mechanization permits the operation of an inertial system at latitudes close to the Polar
Regions, avoiding the singularity associated with the north-slaved mechanization.

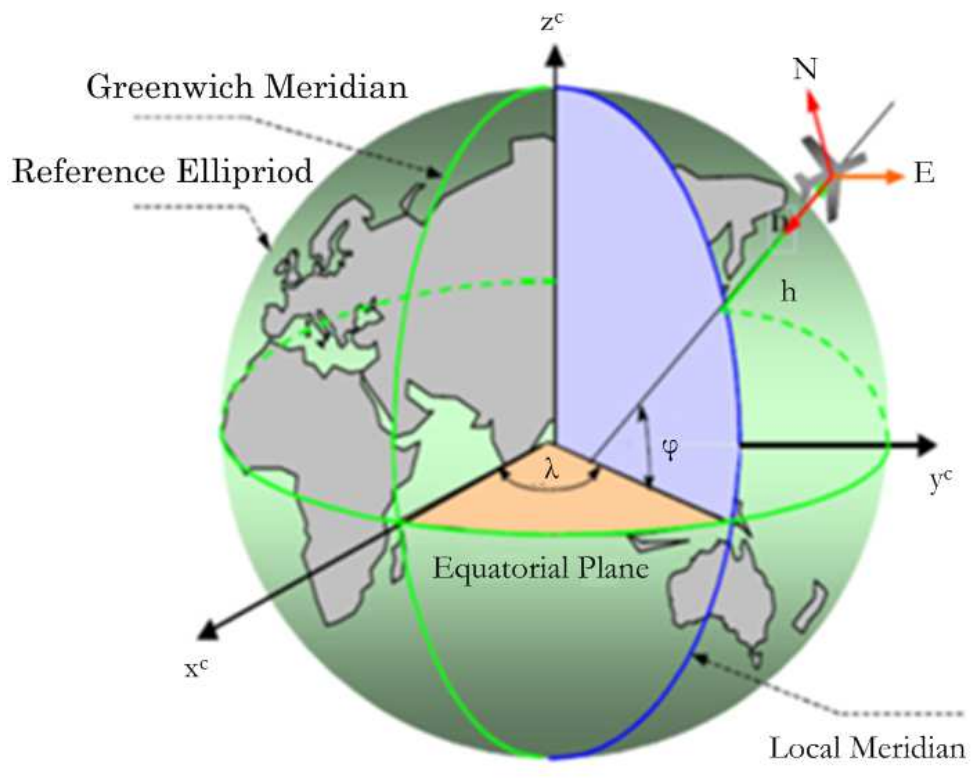

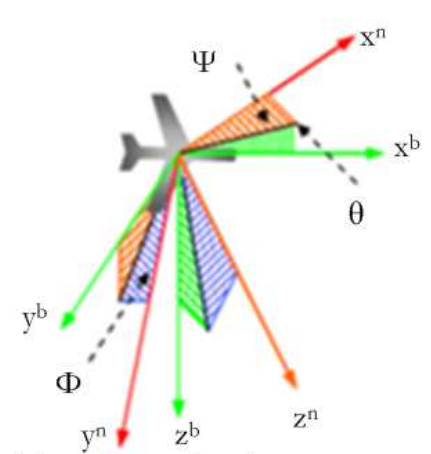

(b) Body and NAV Frames

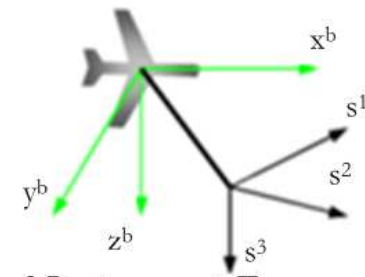

(a) ECEF and NAV Frames

(c) Body and Instrument Frames

Figure 2. Coordinate Systems.

\section{System Flowchart}

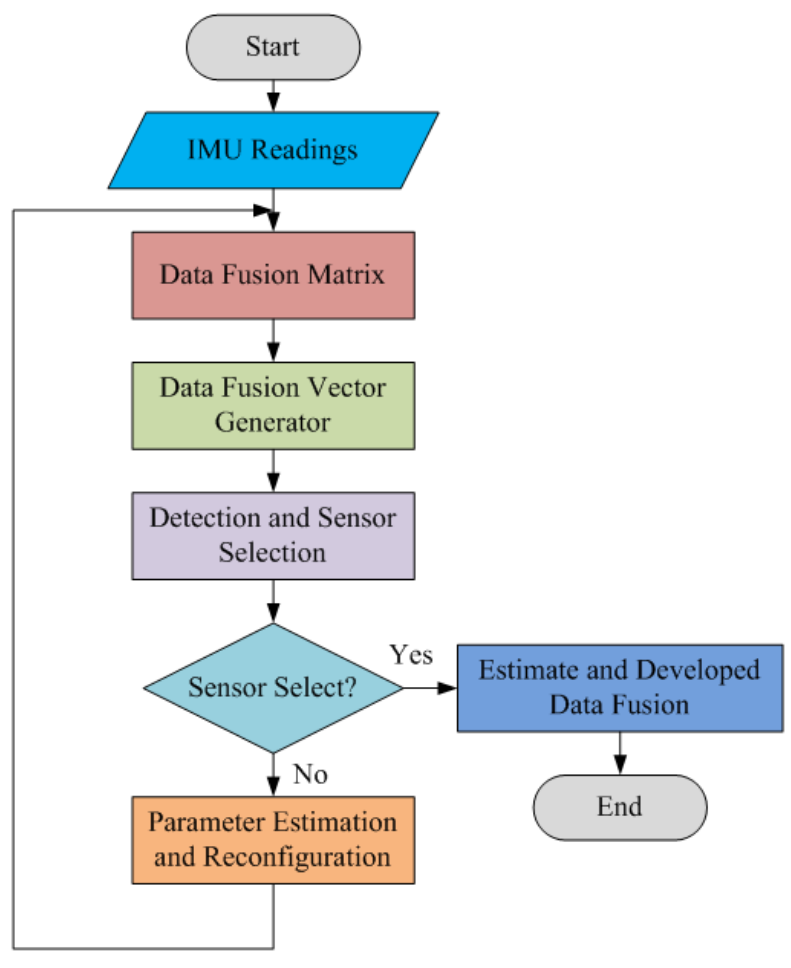

Figure 3. System Flowchart.

The system flowchart for multisensor data fusion algorithm is illustrated in Figure 3. The sensor readings (IMU readings) are declared according to the specified analysis. And then the data fusion matrix for multisensor data fusion algorithm is calculated based on the navigation system of UAV and other spacecraft. The data fusion vector is generated after the calculation process. The user has to select the sensor and it could be detected according to the specified data set from external command. If the sensors are not selected from user the reconfiguration process could be started to the calculation of data fusion process. When the sensors are selected from user, the condition of developed data fusion state and estimate multisensory fusion (MF) is appeared.

\section{Simulation Results}

A. Cover Window

The multi-sensor data fusion algorithm for aircraft navigation system is implemented with the help of MATLAB. The detailed algorithm for implemented system could be examined based on the rules and regulations from the MATLAB GUI creation. The cover window for simulation results for multi-sensor data fusion algorithm is activated when the MATLAB starts. In this result page, there are three main buttons to analyze the simulation of multi-sensor data fusion algorithm. They are OPEN, SENSOR and EXIT. The SENSOR button is the main idea to implement the multi-sensor data fusion algorithm for present system. The EXIT button is to close the overall system simulation from MATLAB environments.

B. Simulation Setup for Multi-sensor Data Fusion

The simulation setup for multi-sensor data fusion algorithm is the major scheme for practical test. In this window, the display GUI and command button for selection of examined. There are a lot of data set and sensors for simulation setup. The exponents, iteration and improvement values are assigned 
by declaration from external user.

According to the simulation setup for multi-sensor data fusion algorithm, the respective simulation results and MF results are mentioned and displayed. The simulation results clearly say the improvement of multi-sensor data fusion algorithm for aircraft navigation for UAV developments. The sensor management and multi-sensor data fusion algorithm are much related to implement based on the idea for control analysis approach and stability analysis for UAV or spacecraft system. Based on the user identification from external command for multi-sensor data fusion algorithm, the fusion algorithm is very efficient to analyze the specifications for Gyro sensor and accelerometers for UAV.

C. Simulation Result for Data Set 1 with 2 Sensors Condition The simulation result for data set 1 with 2 sensors condition is illustrated in Figure 5.

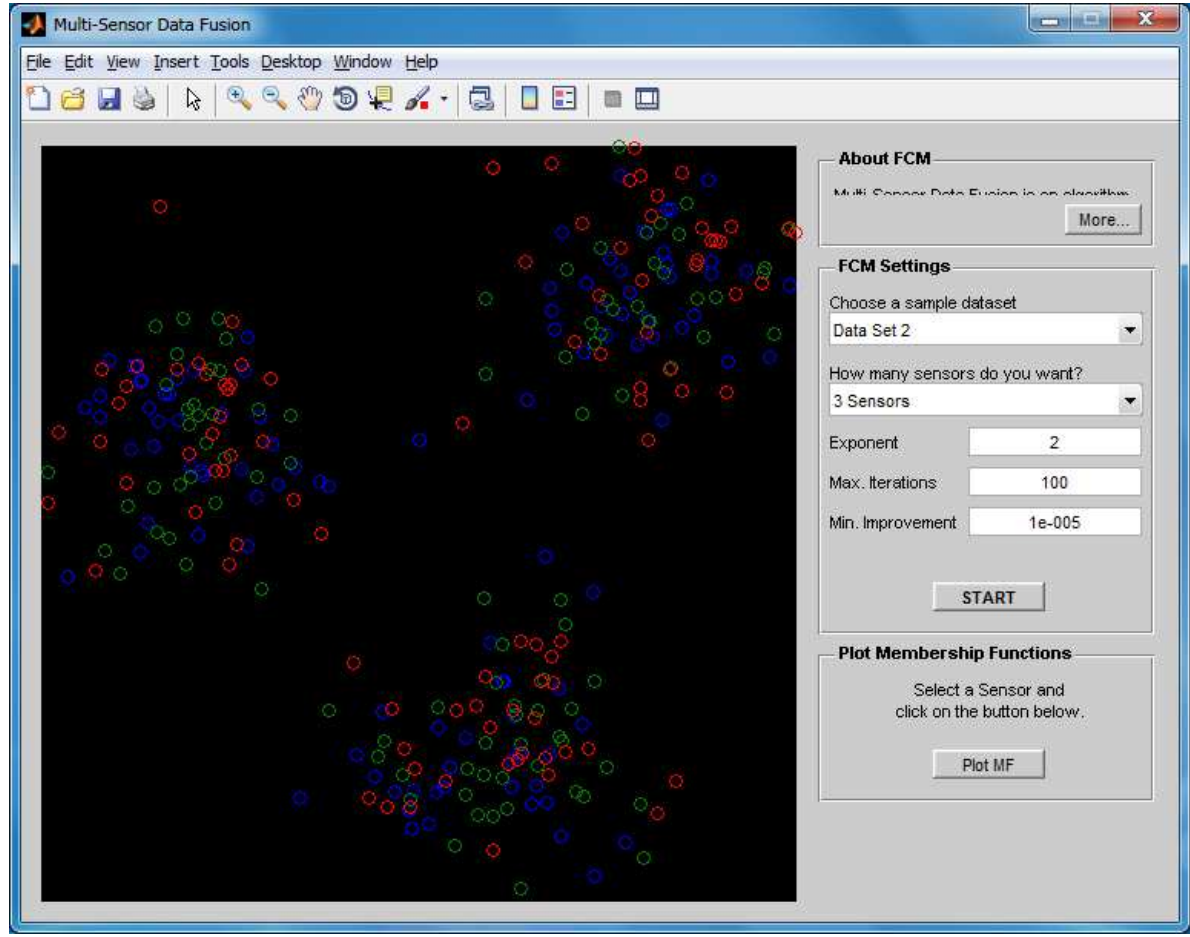

Figure 4. Simulation Setup for Multi-sensor Data Fusion.

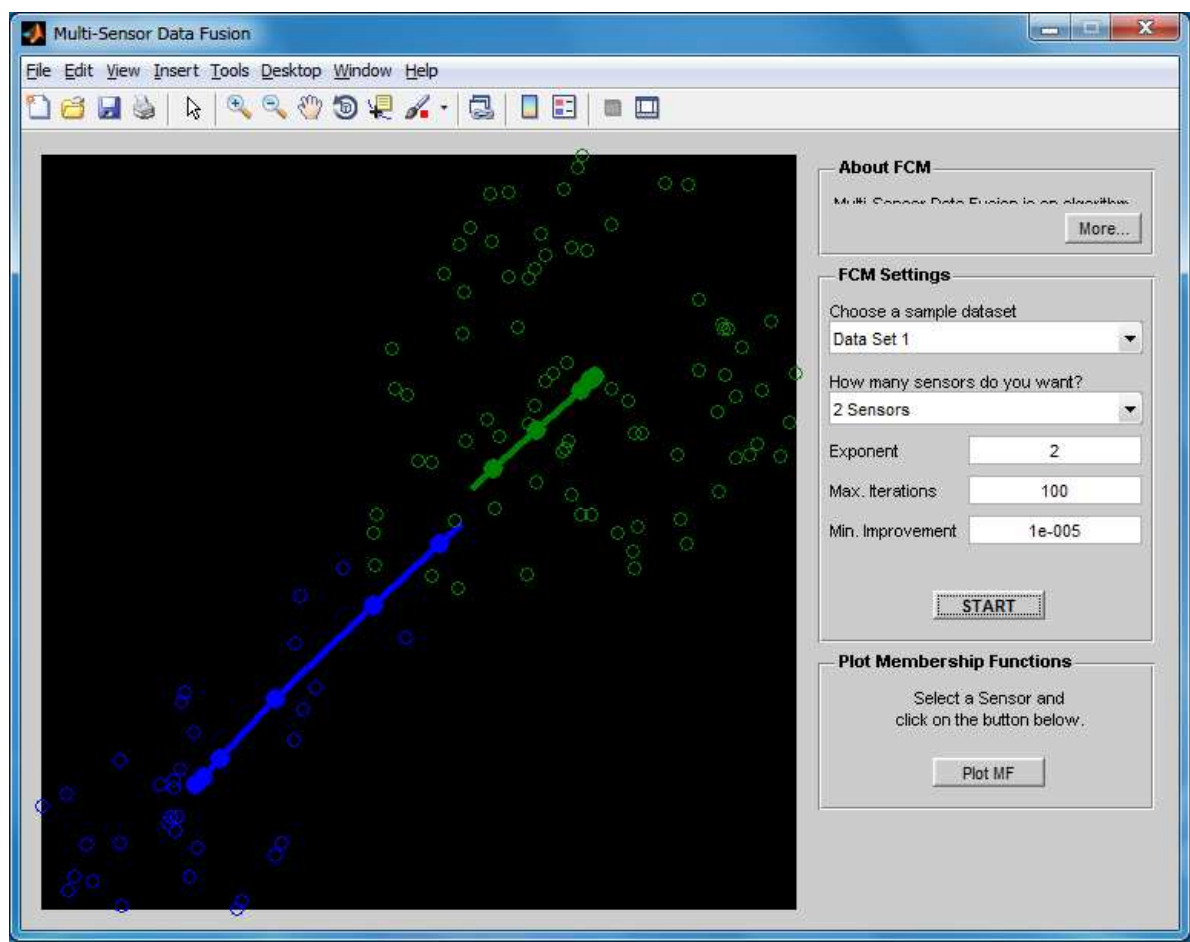

Figure 5. Screenshot Result for Data Set 1 with 2 Sensors Condition. 
This result says the two paths of sensor fusion which indicates the blue and green color paths are easily examined for sensor management by selecting the MF point from external point selected. Based on the selection point, the multi-sensor fusion (MF) result for data set 1 with 2 sensors condition is displayed in Figure 6.

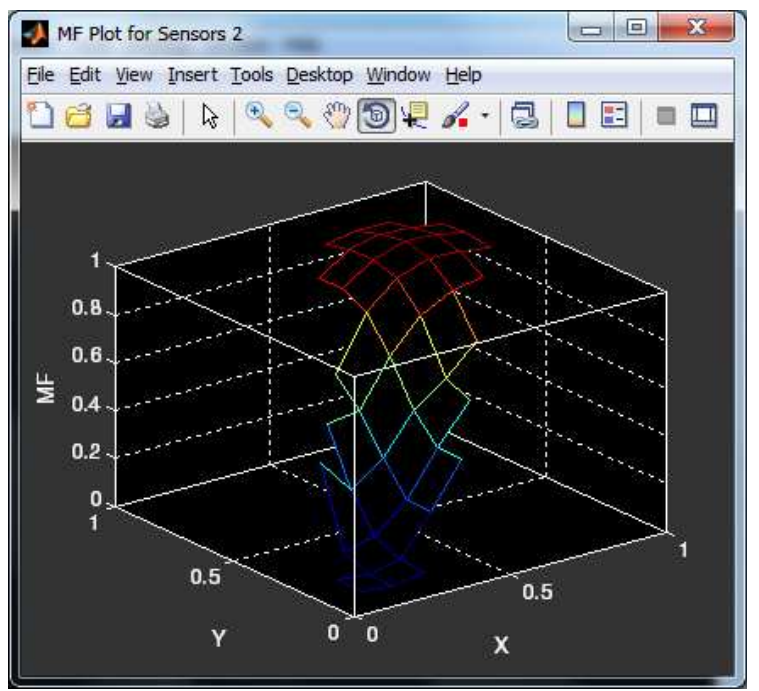

Figure 6. Multi-sensor Fusion (MF) Result for Data Set 1 with 2 Sensors Condition.

D. Simulation Result for Data Set 5 with 7 Sensors Condition.

The simulation result for data set 5 with 7 sensors condition is distinguished in Figure 7. This result enunciates the seven paths of sensor fusion paths are easily inspected for sensor management by selecting the MF point from external point chosen. Based on the selection point, the multi-sensor fusion (MF) result for data set 5 with 7 sensors condition is nearby in Figure 8.

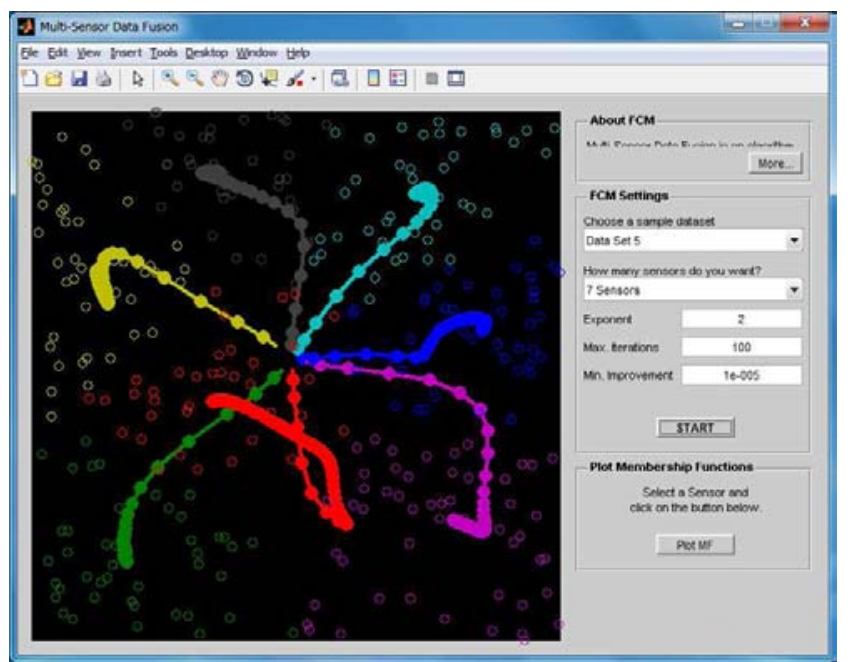

Figure 7. Screenshot Result for Data Set 5 with 7 Sensors Condition.

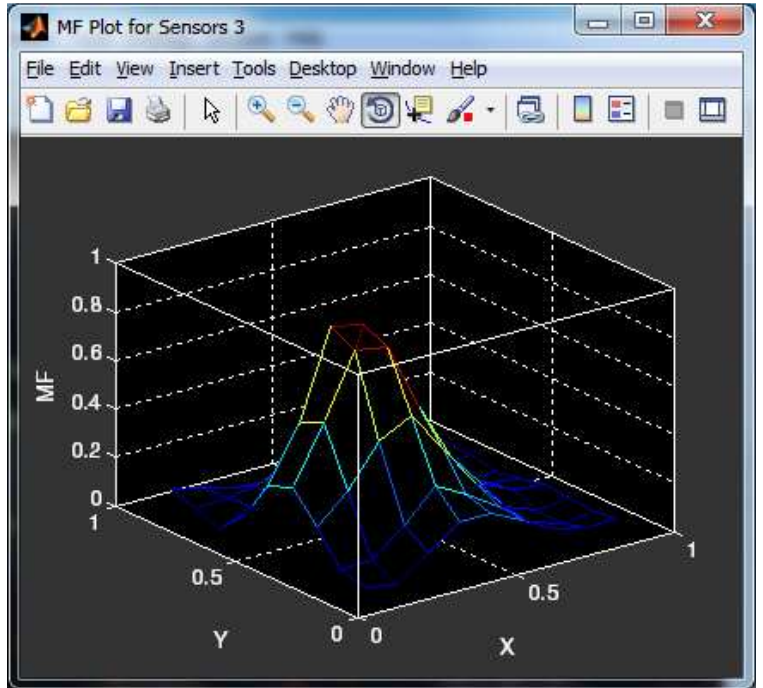

Figure 8. Multi-sensor Fusion (MF) Result for Data Set 5 with 7 Sensors Condition.

\section{Conclusion}

The process of algorithm selection and systems integration is complete and the relevant equations presented using a common nomenclature. A simulation has been created to support generic target and sensor models stimulating multiple-model target tracking, data up-link, and missile state observer. The estimates of target and missile data stimulate conventional guidance laws and trajectory optimization. The process of model verification, technique validation and tuning is in progress and some initial results from this program are presented. A simulation infrastructure has been created capable of supporting any model requiring state integration. For transportability, and compatibility with a range of host systems, it was developed as a self-contained entity. This infrastructure is used on several programs for terrain following navigation, transfer alignment, and pre-launch studies involving air-launched munitions from various fighter aircraft. This expanding user group has accelerated software verification, and the refinement of the facilities provided.

\section{References}

[1] RAO B. S., and DURRANT-WHYTE H. F., (September 1991). "Fully decentralized algorithm for multisensor Kalman filtering," IEE PROCEEDINGS-D, Vol. 138, No. 5, pp. 413-420.

http://portal.acm.org/citation.cfm?id=942356\&dl=ACM\&coll $=$ portal.

[2] PAO L. Y., and BALTZ N. T. (June 1999). "Control of Sensor Information in Distributed Multisensor Systems," Proc. American Control Conference, San Diego, CA, pp. 2397-2401.

[3] DURRANT-WHYTE H. F., and LEONARD J. J., (13-18 May 1990). "Toward a fully decentralized architecture for multi-sensor data fusion," Robotics and Automation, 1990. Proceedings., IEEE International Conference, Vol. 2, pp. 1331 -1336 . 
[4] DURRANT-WHYTE H. F., RAO B. Y. S., and HU H., (4 Feb 1991). "Toward a fully decentralized architecture for multi-sensor data fusion," Principles and Applications of Data Fusion, IEE Colloquium, pp. 2/1 - 2/4.

[5] Jiang Dong, Dafang Zhuang, Yaohuan Huang and Jingying Fu, "Advances in Multi-Sensor Data Fusion: Algorithms and Applications", Sensors 2009, 9, 7771-7784; doi: 10.3390/s91007771.

[6] Mohammad Amin Ahmad Akhoundi, Ehsan Valavi, "Multi-Sensor Fuzzy Data Fusion Using Sensors with Different Characteristics", The CSI Journal on Computer Science and Engineering (JCSE), submitted.

[7] Hugh Durrant-Whyte, "Multi Sensor Data Fusion", Australian Centre for Field Robotics The University of Sydney NSW 2006, Australia, January 22, 2001.

[8] S. A. Quadri, Othman Sidek, "Optimization and Comparison of Two Data Fusion Algorithms for an Inertial Measurement Unit", International Journal of Computer Science Engineering (IJCSE), Vol. 2 No. 04 July 2013.

[9] Y. A. Vershinin,"A Data Fusion Algorithm for Multisensor Systems", ISIF, 2002.
[10] Jitendra R. Raol, "Multi-Sensor Data Fusion with MATLAB", CRC Press Taylor \& Francis Group, 2010.

[11] Joseph S. J. Peri, "Approaches to Multisensor Data Fusion", JOHNS HOPKINS APL TECHNICAL DIGEST, VOLUME 22, NUMBER 4 (2001).

[12] Sandra Rodríguez-Valenzuela, Juan A. Holgado-Terriza, José M. Gutiérrez-Guerrero and Jesús L. Muros-Cobos, "Distributed Service-Based Approach for Sensor Data Fusion in IoT Environments", Sensors 2014, 14, 19200-19228; doi: 10.3390/s141019200.

[13] KS Nagla, Moin Uddin, and Dilbag Singh, "Multisensor Data Fusion and Integration for Mobile Robots: A Review", International Journal of Robotics and Automation (IJRA) Vol. 3, No. 2, June 2014, pp. 131 138.

[14] Sajjad Safari, Faridoon Shabani, Dan Simon, "Multirate multisensor data fusion for linear systems using Kalman filters and a neural network", Aerospace Science and Technology 39 (2014) 465-471. 